\title{
DMFT Reveals the Non-Hermitian Topology and Fermi Arcs in Heavy-Fermion Systems
}

\author{
Yuki Nagai® ${ }^{1,2}$ Yang Qi®, ${ }^{3,2}$ Hiroki Isobe, ${ }^{2}$ Vladyslav Kozii $\odot,{ }^{4,5,2}$ and Liang $\mathrm{Fu}^{2}$ \\ ${ }^{1}$ CCSE, Japan Atomic Energy Agency, 178-4-4, Wakashiba, Kashiwa, Chiba 277-0871, Japan \\ ${ }^{2}$ Department of Physics, Massachusetts Institute of Technology, Cambridge, Massachusetts 02139, USA \\ ${ }^{3}$ Department of Physics, Fudan University, Shanghai 200433, China \\ ${ }^{4}$ Department of Physics, University of California, Berkeley, California 94720, USA \\ ${ }^{5}$ Materials Sciences Division, Lawrence Berkeley National Laboratory, Berkeley, California 94720, USA
}

(Received 27 September 2019; revised 23 October 2020; accepted 26 October 2020; published 25 November 2020)

\begin{abstract}
When a strongly correlated system supports well-defined quasiparticles, it allows for an elegant onebody effective description within the non-Hermitian topological theory. While the microscopic many-body Hamiltonian of a closed system remains Hermitian, the one-body quasiparticle Hamiltonian is nonHermitian due to the finite quasiparticle lifetime. We use such a non-Hermitian description in the heavyfermion two-dimensional systems with the momentum-dependent hybridization to reveal a fascinating phenomenon which can be directly probed by the spectroscopic measurements, the bulk "Fermi arcs." Starting from a simple two-band model, we first combine the phenomenological approach with the perturbation theory to show the existence of the Fermi arcs and reveal their connection to the topological exceptional points, special points in the Brillouin zone where the Hamiltonian is nondiagonalizable. The appearance of such points necessarily requires that the electrons belonging to different orbitals have different lifetimes. This requirement is naturally satisfied in the heavy-fermion systems, where the itinerant $c$ electrons experience much weaker interaction than the localized $f$ electrons. We then utilize the dynamical mean field theory to numerically calculate the spectral function and confirm our findings. We show that the concept of the exceptional points in the non-Hermitian quasiparticle Hamiltonians is a powerful tool for predicting new phenomena in strongly correlated electron systems.
\end{abstract}

DOI: 10.1103/PhysRevLett.125.227204

Introduction.-A standard theory of noninteracting metals and semimetals in two dimensions assumes that the Fermi surface is represented by the closed lines or isolated points, respectively, defining the transport and spectroscopic properties. In the presence of disorder or strong correlations, however, the situation is much more delicate. For instance, disorder in graphene induces the finite density of states at the nodal point leading to nonzero conductivity $[1,2]$. Other examples include the observation of the quantum oscillations in the small gap Kondo insulators $\mathrm{SmB}_{6}[3,4]$ and $\mathrm{YbB}_{12}[5,6]$, which is canonical evidence for a finite Fermi surface. Finally, the pseudogap phase of the hole-doped cuprate high-temperature superconductors exhibits the Fermi arcs, opened Fermi surfaces with endpoints [7], which indicate to the strong correlations in the system and remain a matter of intense theoretical discussion [8-11]. All these examples clearly demonstrate that interactions in the correlated materials may have much more drastic effect than simple renormalization of the bare parameters of the band structure.

Recently, it was proposed that the nontrivial structure of the Fermi surface in some cases can be explained within the non-Hermitian topological theory [12-14]. This theory naturally generalizes the topological description of quantum matter, e.g., topological insulators and semimetals, to the finite-temperature regime when quasiparticles experience scatterings due to disorder or interactions. While the original many-body Hamiltonian is Hermitian, the effective one-body quasiparticle Hamiltonian becomes nonHermitian because of the finite quasiparticle lifetime. Such a description shows, in particular, that the presence of disorder at zero temperature [14] or interactions at finite temperature [13] generically stretchs the nodal points in two-dimensional Dirac materials into the open bulk Fermi arcs, provided the lifetimes of fermions from different orbitals are different. The origin of the arcs is intimately related to the presence of an interesting topological object, "exceptional points" (EPs) in the Brillouin zone, at which the Hamiltonian becomes nondiagonalizable [15]. These points are the direct consequence of the non-Hermitian description of an interacting system in terms of the singleparticle Hamiltonian. Importantly, the EPs are topologically stable, implying that the Fermi arcs cannot be eliminated by small perturbations $[12,13]$.

In this work, we study how the non-Hermitian topology reveals itself in the two-dimensional heavy fermion compounds. Different types of electrons naturally have different lifetimes in these materials, since the localized $f$ electrons experience much stronger electron-electron interaction than the itinerant $c$ electrons. This observation makes heavy 
fermions a perfect platform for searching for the EPs and Fermi arcs. More specifically, we consider a Kondo semimetal, which is understood as a Kondo insulator with a small momentum-dependent hybridization gap that has nodes on the Fermi surface. Conventional Kondo insulators exhibit insulating behavior at low temperature because of the hybridization at the Fermi energy, which crosses over to a metallic regime with the $c$-electron Fermi surface as the temperature raises [16]. In contrast, as we demonstrate below, different points of the (nonhybridized) Fermi surface in a Kondo semimetal with a momentum-dependent gap undergo the crossover at different temperatures, leading eventually to the Fermi arcs which connect the EPs.

To describe a heavy-fermion system, we use the periodic Anderson model (PAM) [17] in two dimensions with a momentum-dependent ( $d$-wave) hybridization gap. First, using the phenomenological approach combined with the perturbation theory, we show that the difference between the electron correlations in $c$ and $f$ bands naturally leads to two different lifetimes, resulting in EPs and Fermi arcs at certain temperatures. To confirm our results numerically, we further adopt the dynamical mean field theory (DMFT) with the numerically exact continuous-time quantum Monte Carlo solver. The spectral function calculated by the DMFT clearly shows that there are Fermi arcs defined as the lines in the Brillouin zone where the spectral function has a peak at zero energy. We suggest that these Fermi arcs may be directly observed by angle-resolved photoemission spectroscopy (ARPES) in the small-gap Kondo lattices such as CeNiSn [18].

Non-Hermitian quasiparticle Hamiltonian.-The nonHermitian Hamiltonian of the finite-lifetime quasiparticles $H(\boldsymbol{k}, \omega)$ is conveniently defined through the retarded electron Green's function $G^{R}(\boldsymbol{k}, \omega)[12,13]$ :

$$
H(\boldsymbol{k}, \omega) \equiv\left[\omega-G^{R}(\boldsymbol{k}, \omega)\right]^{-1}=H_{0}(\boldsymbol{k})+\Sigma(\boldsymbol{k}, \omega),
$$

where $H_{0}(\boldsymbol{k})$ is the bare (noninteracting) Hamiltonian and $\Sigma(\boldsymbol{k}, \omega)$ is the electron's self-energy that includes the effect of the electron-electron interaction. Note that the quasiparticle Hamiltonian $H(\boldsymbol{k}, \omega)$ can be non-Hermitian with the complex spectrum $\mathcal{E}_{n}(\boldsymbol{k}, \omega)$. In general, $\Sigma$ is a sum of the Hermitian part $\Sigma^{\prime}$ and the non-Hermitian part $\Gamma$ : $\Sigma=\Sigma^{\prime}-i \Gamma . \Sigma^{\prime}$ renormalizes the bare band structure, while $\Gamma$ leads to finite quasiparticle lifetimes. The spectrum $\mathcal{E}_{n}(\boldsymbol{k}, \omega)$ determines the complex poles $\omega=E_{n}(\boldsymbol{k})$ of the Green's function according to $E_{n}=\mathcal{E}_{n}\left(\boldsymbol{k}, E_{n}\right)$. In the vicinity of a first-order pole, the Green's function takes the form $G^{R}(\boldsymbol{k}, \omega) \sim\left[1 / \omega-E_{n}(\boldsymbol{k})\right]$. The real part of $E_{n}(\boldsymbol{k})$ determines the quasiparticle's dispersion, while its imaginary part defines the quasiparticle's inverse lifetime.

The important difference between Hermitian and nonHermitian Hamiltonians is that the latter can be nondiagonalizable at certain momenta. Those points are called "exceptional points" in the mathematical physics literature [15]. At the EPs, linearly independent eigenstates do not span the full Hilbert space. One of the authors showed that these EPs in two and higher dimensions are topologically stable [12]. Topological EPs can appear in the quasiparticle spectrum of zero- and small-gap materials such as Dirac semimetals in two dimensions, provided the electrons corresponding to the different orbitals have different lifetimes [13]. We show that this scenario is also realized in the heavy fermion systems with the momentumdependent hybridization gap, where the two orbitals correspond to the itinerant $c$ and localized $f$ electrons [19-21].

To study heavy-fermion materials, we consider the PAM defined as

$$
\mathcal{H}=\sum_{\boldsymbol{k} \sigma \sigma^{\prime}}\left(\begin{array}{ll}
f_{\boldsymbol{k} \sigma}^{\dagger} & c_{\boldsymbol{k} \sigma}^{\dagger}
\end{array}\right)\left[H_{0}(\boldsymbol{k})\right]_{\sigma \sigma^{\prime}}\left(\begin{array}{c}
f_{\boldsymbol{k} \sigma^{\prime}} \\
c_{\boldsymbol{k} \sigma^{\prime}}
\end{array}\right)+\mathcal{H}_{\mathrm{int}},
$$

with the lattice Hamiltonian

$$
\left[H_{0}(\boldsymbol{k})\right]_{\sigma \sigma^{\prime}}=\delta_{\sigma \sigma^{\prime}}\left(\begin{array}{cc}
\epsilon_{f \boldsymbol{k}} & v_{\boldsymbol{k}} \\
v_{\boldsymbol{k}} & \epsilon_{c \boldsymbol{k}}
\end{array}\right),
$$

and the interaction term $\mathcal{H}_{\text {int }}=U \sum_{i} f_{i \uparrow}^{\dagger} f_{i \uparrow} f_{i \downarrow}^{\dagger} f_{i \downarrow}$. Here, $c_{k \sigma}\left(f_{k \sigma}\right)$ is an annihilation operator for an itinerant $c$ (localized $f$ ) electron with momentum $\boldsymbol{k}$ and spin $\sigma$, with dispersions $\epsilon_{c k}=-2 t\left(\cos k_{x}+\cos k_{y}\right)-\mu$ and $\epsilon_{f k}=$ $2 t_{f}\left(\cos k_{x}+\cos k_{y}\right)+\epsilon_{f 0}-\mu$, respectively. We use $t$ as the unit of the energy scale hereafter, and $\mu$ here is the chemical potential. We consider the case of half-filling, which corresponds to $\mu=0$ and $\epsilon_{f 0}=-U / 2$, and is characterized by the particle-hole symmetry. At half-filling, the term $\epsilon_{f 0}=-U / 2$ is exactly canceled by the Hartree part of the electron's self-energy, so we omit it for the rest of the Letter for brevity. The hybridization gap is expressed as $v_{k}=v$ for the $s$-wave case which describes the Kondo insulators and $v_{k}=v\left(\cos k_{x}-\cos k_{y}\right)$ for the $d$-wave case which corresponds to the Kondo semimetals, respectively. In this work, we focus on the $d$-wave gap, which has point nodes on the (unhybridized) Fermi surface at $\left(k_{x}, k_{y}\right)=$ $( \pm \pi / 2, \pm \pi / 2)[22]$.

It is known that the PAM exhibits a number of strongly correlated phenomena showing the non-Fermi-liquid behavior [25] and undergoing the Mott transition [26-28] near some particular fillings. In our work, we stay away from these special fillings, i.e., in the regime where the Fermi liquid description remains adequate.

Because of the fact that the $c$ electrons in model Eq. (2) are not interacting, only $f$ electrons acquire a nonzero selfenergy, leading to

$$
[\Sigma(\boldsymbol{k}, \omega)]_{\sigma \sigma^{\prime}}=\left(\begin{array}{cc}
\Sigma^{f}(\boldsymbol{k}, \omega) \delta_{\sigma \sigma^{\prime}} & 0 \\
0 & 0
\end{array}\right)
$$

and resulting in different lifetimes for different orbitals. This point is explained in detail in Sec. S2 of the Supplemental 


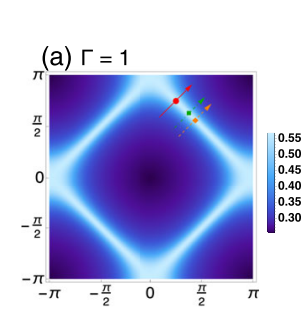

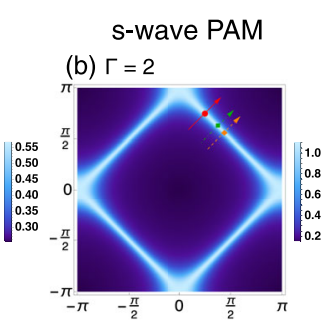

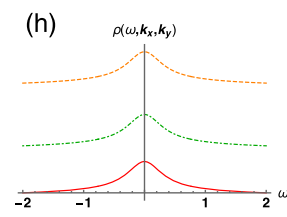

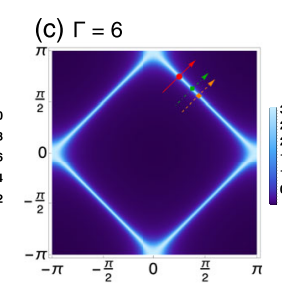

(i)

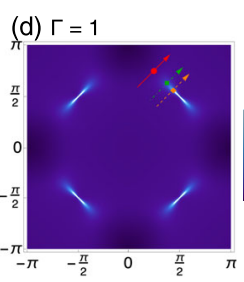

(j)

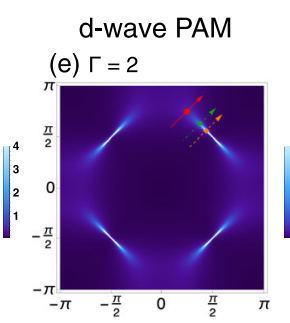

(k)

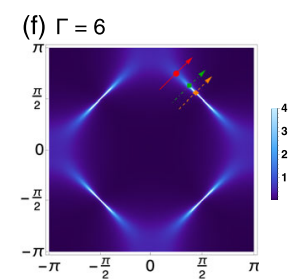

(I)
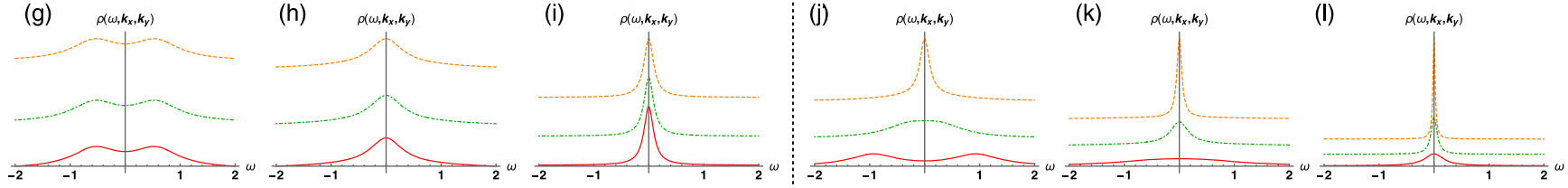

$(\pi / 4,3 \pi / 4)$

$(3 \pi / 8,5 \pi / 8)$

$(7 \pi / 16,9 \pi / 16)$

FIG. 1. (a)-(f) Momentum dependence of the zero-energy spectral functions $\rho\left(\omega=0+i \eta, k_{x}, k_{y}\right)$ extracted from Eq. (7). We set $v=0.75, t=1, t_{f}=0$, and $\eta=0.01$. The red circle, green square, and orange diamond denote points at $\left(k_{x}, k_{y}\right)=(\pi / 4,3 \pi / 4)$, $(3 \pi / 8,5 \pi / 8)$, and $(7 \pi / 16,9 \pi / 16)$, respectively. (g)-(l) Energy dependence of the spectral function $\rho\left(\omega, k_{x}, k_{y}\right)$ at these points in momentum space. The results for the $s$-wave periodic Anderson model (PAM) are shown in (a)-(c) and (g)-(i). The results for the $d$-wave PAM are shown in (d)-(f) and (j)-(l). The $f$-electron inverse lifetime $\Gamma$ is fixed to $\Gamma=1$ for (a), (g), (d), (e), $\Gamma=2$ for (b), (h), (e), (k), and $\Gamma=6$ for (c), (i), (f), (l).

Material (SM) [22]. Thus, the two-lifetime description naturally arises in the PAM.

The $f$-electron self-energy is computed using a secondorder perturbation theory in Sec. S2 of the SM [22] (see also, Ref. [29]). At temperatures comparable to or higher than the hybridization scale $v$ we can ignore the momentum dependence of $\Sigma(\omega)$. This is because the finite-temperature broadening renders the $f$-band approximately flat. Hence, the on site interaction and a flat band together result in a local self-energy. Since we are interested in the spectrum of the low-energy electron excitations, we focus on the lowfrequency behavior of the self-energies. In particular, in the zero-frequency limit, $\Sigma^{f}$ can be treated as a purely imaginary constant, $\Sigma^{f}(\boldsymbol{k}, 0)=-i \Gamma$, while the real part vanishes because the particle-hole symmetry pins the energy of the $f$ electron to zero. Furthermore, at small frequencies, one can expand $\Sigma^{f}$ in the Taylor series:

$$
\Sigma^{f}(\omega)=-a_{1} \omega-i\left(\Gamma+a_{2} \omega^{2}\right)+\cdots
$$

Our DMFT calculations presented later show that coefficients $a_{1}$ and $a_{2}$ are generally positive. The inclusion of the positive higher-order terms $a_{1}$ and $a_{2}$ does not change the qualitative features of the electron spectrum discussed next. In particular, the effect of including $a_{1}$, which is the leading term of the real part of $\Sigma^{f}(\omega)$, is the renormalization of certain band parameters: it renormalizes $t_{f}$ to $Z t_{f}, \Gamma$ to $Z \Gamma$, and $v_{k}$ to $Z^{1 / 2} v_{k}$, respectively, while keeping $t$ unaffected. (This is analyzed in detail in Sec. S2 of the SM.) Here, $Z \equiv\left(1+a_{1}\right)^{-1}$.

Exceptional points and bulk Fermi arcs.-We now discuss the electron spectral function and demonstrate the Fermi arcs in the $d$-wave PAM. The spectral function derived from Eq. (1) is shown in Fig. 1.
In general, the inverse lifetime $\Gamma$ is small at low temperatures and large at high temperatures, reflecting a long and a short quasiparticle lifetime, respectively. Hence, we demonstrate low-temperature and high-temperature phenomena using $\Gamma=1, \Gamma=2$, and $\Gamma=6$, respectively. In the $s$-wave PAM, there is an isotropic gap in the whole momentum space as shown in Figs. 1(a) and 1(g). With increasing $\Gamma$, the gap is closed on an entire Fermi surface. This is the standard crossover from a Kondo insulator at low temperatures to a metallic state with a $c$-electron Fermi surface at high temperatures. In the $d$-wave PAM, on the contrary, we can clearly see the bulk Fermi arcs at low temperatures, as shown in Figs. 1(d) and 1(j). The Fermi arcs grow into a full $c$-electron Fermi surface at high temperatures.

The Fermi arcs observed in the $d$-wave PAM can be explained as follows. With $\Sigma=-i \Gamma-a_{1} \omega$, the complex poles of the Green's function are given by

$$
\begin{aligned}
E_{ \pm}(\boldsymbol{k})= & \pm \frac{Z}{2} \sqrt{\left(M_{\boldsymbol{k}}-i \Gamma\right)^{2}+4 Z^{-1}\left|v_{\boldsymbol{k}}\right|^{2}} \\
& +\frac{Z}{2}\left(\epsilon_{f \boldsymbol{k}}-i \Gamma+Z^{-1} \epsilon_{c \boldsymbol{k}}\right)
\end{aligned}
$$

with $M_{k} \equiv \epsilon_{f k}-Z^{-1} \epsilon_{c k}$. This complex spectrum is similar to that for the Dirac semimetals in two dimensions if we replace $M_{\boldsymbol{k}}$ and $\left|v_{\boldsymbol{k}}\right|$ with $v_{x} k_{x}$ and $v_{y} k_{y}$, respectively (and assuming $Z=1$ ) [13].

The spectral function $\rho(\omega, \boldsymbol{k}) \equiv-(1 / \pi) \operatorname{Im} \operatorname{Tr}\left(G^{R}-G^{A}\right)$, with $G^{A} \equiv\left[G^{R}\right]^{\dagger}$, is given by

$$
\rho(\omega, \boldsymbol{k})=-\frac{2}{\pi} \operatorname{Im}\left[\frac{A_{k}}{\omega-E_{+}(\boldsymbol{k})}+\frac{B_{k}}{\omega-E_{-}(\boldsymbol{k})}\right],
$$


with

$$
\begin{aligned}
A_{k} & =\frac{(1+Z) E_{+}(\boldsymbol{k})-Z\left(\epsilon_{c k}+\epsilon_{f \boldsymbol{k}}-i \Gamma\right)}{E_{+}(\boldsymbol{k})-E_{-}(\boldsymbol{k})}, \\
B_{\boldsymbol{k}} & =\frac{Z\left(\epsilon_{c \boldsymbol{k}}+\epsilon_{f \boldsymbol{k}}-i \Gamma\right)-(1+Z) E_{-}(\boldsymbol{k})}{E_{+}(\boldsymbol{k})-E_{-}(\boldsymbol{k})} .
\end{aligned}
$$

The form factors $A_{k}$ and $B_{k}$ originate from the nonzero real part of the self-energy and both equal to 1 in the case when $a_{1}=0$.

We see that the spectral function is given by the sum of two Lorentzians, whose locations and widths are given by the real and imaginary parts of $E_{ \pm}$, respectively. In particular, we focus on the line $\epsilon_{c k}=\epsilon_{f k}=0$, which is the location of the high-temperature $c$-electron Fermi surface. Along this line, Eq. (6) is simplified to the following form:

$$
E_{ \pm}(\boldsymbol{k})= \pm \frac{Z}{2} \sqrt{-\Gamma^{2}+4 Z^{-1}\left|v_{\boldsymbol{k}}\right|^{2}}+Z \epsilon_{f \boldsymbol{k}}-i Z \frac{\Gamma}{2} .
$$

We first discuss how the temperature-dependent $\Gamma$ affects the electron spectrum and spectral function at a fixed momentum $\boldsymbol{k}$ at the unhybridized Fermi surface, $\epsilon_{c k}=\epsilon_{f k}=0$. For simplicity, we consider the case $Z=1$ $\left(a_{1}=0\right)$. In general, we expect $\Gamma$ to increase with temperature and approach zero in the $T=0$ limit. The competition between the temperature-dependent $\Gamma$ and the hybridization $v_{k}$ can explain the Kondo crossover. At low temperatures, when $4\left|v_{k}\right|^{2}>\Gamma^{2}, E_{ \pm}$have different real parts, and the spectral function is a superposition of two peaks. The energy difference between the two peaks, $\sqrt{4\left|v_{k}\right|^{2}-\Gamma^{2}}$, represents a hybridization gap. This indicates that the $f$ and $c$ electrons are hybridized, and the $f$ electrons join the $c$ electrons to contribute to the volume of the Fermi surface. At high temperature, when $4\left|v_{k}\right|^{2}<\Gamma^{2}, E_{ \pm}$have the same real part, and the spectral function exhibits a single peak located at $\omega=0$. This indicates that there is no gap, and corresponding momenta $\boldsymbol{k}$ form a Fermi surface.

For the $s$-wave PAM, the crossover between the two behaviors happens simultaneously for all $\boldsymbol{k}$ at the temperature corresponding to $\Gamma=2 v$, accompanied with the emergence of a complete $c$-electron Fermi surface as the temperature raises. For the $d$-wave PAM, in contrast, the crossover for the different points on the Fermi surface occurs at different temperatures determined by $v_{\boldsymbol{k}}$. In particular, for $\Gamma<2 v$, the Fermi surface is separated into two regions by EPs located at $2\left|v_{k}\right|=\Gamma$, where $E_{ \pm}$become degenerate. The hybridization gap then develops only for the momenta satisfying $4\left|v_{k}\right|^{2}>\Gamma^{2}$, while momenta with $4\left|v_{k}\right|^{2}<\Gamma^{2}$ form Fermi arcs. At zero temperature, the system is a Kondo semimetal with point nodes. At finite temperature, the nodal points split into the Fermi arcs which grow longer as the temperature and $\Gamma$ increase,

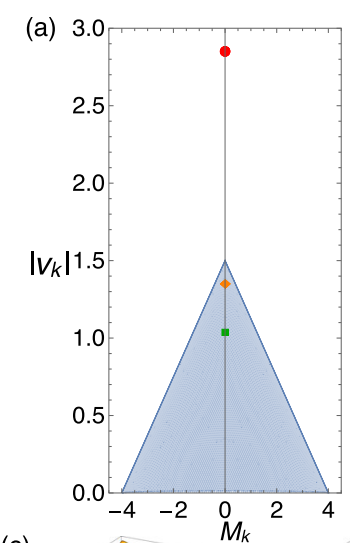

(b)

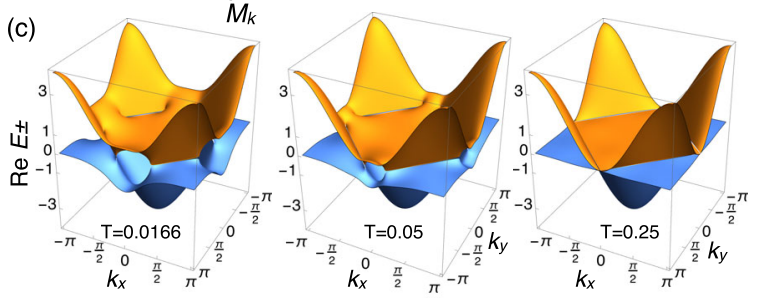

FIG. 2. (a) $\left(M_{k},\left|v_{k}\right|\right)$ space in the two-dimensional model for a Kondo insulator. The shaded region indicates the possible $\left(M_{k},\left|v_{k}\right|\right)$ region. The red circle, orange diamond, and green square denote the value of the lifetime $\Gamma$ at $T=1 / 4,1 / 20$, and $1 / 60$, respectively. The exceptional points (EPs) move from the origin on the $M_{k}$ axis with increasing $\Gamma$. The EPs disappear when they are outside the shaded region. (b) Schematic figures of the EPs. The EPs move from the middle of the line $\epsilon_{c k}=0$ to the corners of the square (unhybridized Fermi surface) as temperature increases. (c) Momentum dependence of the real part of the complex energy spectrum of the non-Hermitian quasiparticle Hamiltonian with different temperatures from $T=1 / 60$ to $T=1 / 4$. The vales of $\Gamma$ are extracted from the DMFT calculation.

until the full $c$-electron Fermi surface emerges at $\Gamma=2 v$, see Fig. 2.

We notice that when the two peaks in the spectral function are closer than the peak width, they become inseparable experimentally. Hence, the precise identification of the ending points of the Fermi arcs may strongly depend on the details of the data analysis, not necessarily giving the exact position of the EPs [22]. However, despite this limitation of the spectroscopy methods, the existence of the Fermi arcs is profoundly tied to the EPs in the quasiparticle Hamiltonian.

Dynamical mean field theory.-To treat the heavy fermion systems more accurately, we use the dynamical mean field theory (DMFT). In the DMFT, we assume that the self-energy does not depend on the momentum:

$$
\Sigma^{f}(\boldsymbol{k}, \omega)=\Sigma^{f}(\omega) .
$$

This assumption is appropriate when the temperature is not too low compared to the maximum of the hybridization energy [22]. The momentum dependence does not affect 

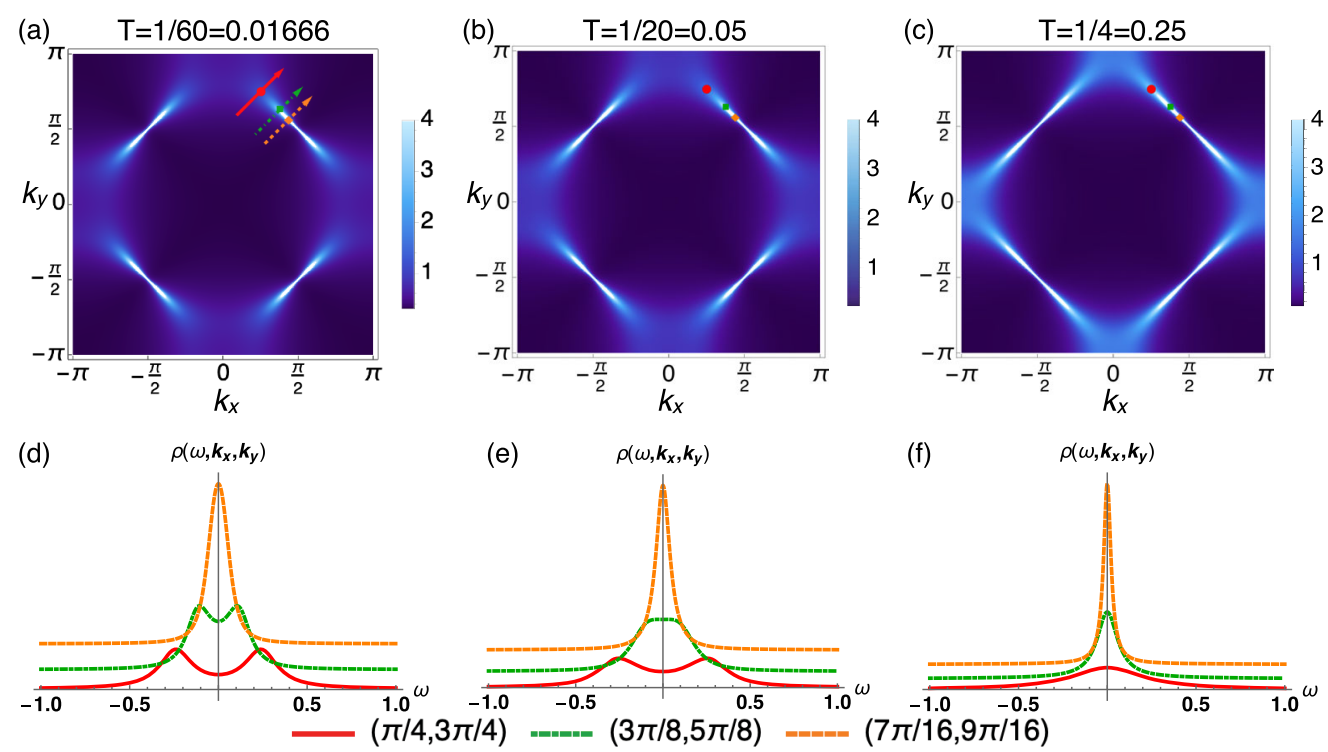

FIG. 3. (a)-(c) Momentum dependence of the zero-energy spectral functions $\rho\left(\omega=0, k_{x}, k_{y}\right)$ obtained by the DMFT calculation. The red circle, green square, and orange diamond denote points at $\left(k_{x}, k_{y}\right)=(\pi / 4,3 \pi / 4),(3 \pi / 8,5 \pi / 8)$, and $(7 \pi / 16,9 \pi / 16)$, respectively. (d)-(f) Energy dependence of the spectral functions $\rho\left(\omega, k_{x}, k_{y}\right)$ at these points in momentum space. Temperatures are (a),(d) $T=1 / 60$, (b),(e) $T=1 / 20$, and (c),(f) $T=1 / 4$, respectively. For these temperatures, the fitting of the DMFT data gives us $\Gamma=2.06,2.71$, and 5.71, correspondingly. We set $t=1, v=0.75, t_{f}=0$, and $U=8$.

the appearance of the bulk Fermi arcs. We set $v=0.75$ and $t_{f}=0$. To calculate the local and momentum-dependent spectral functions $\rho(\omega)$ and $\rho(\omega, \boldsymbol{k})$, we utilize the DMFT with the numerically exact segment-based hybridizationexpansion continuous-time quantum Monte Carlo impurity solver (CT-HYB) [30]. We point out that there is no fermion sign problem since the off-diagonal elements of the self-energy in the spin space are zero in our two-orbital system [31]. After confirming that the self-energy for $c$ electrons is zero in the two-orbital DMFT calculation, we use the one-orbital DMFT calculation with integrating out the $c$-electron degrees of freedom. Here, the effective impurity problem is solved by an open-source program package, iQIST [32]. We set $U=8$.

Figure 3 shows the spectral functions and the Fermi arcs. To calculate the real-frequency self-energy $\Sigma(\omega)$ and the spectral function $\rho(\omega, \boldsymbol{k})$, we use the Padé approximation as the method for the numerical analytic continuation of the self-energy $\Sigma\left(i \omega_{n} \rightarrow \omega\right)$. After using the Padé approximation, we fit the self-energy with the polynomial $\Sigma_{f}(\omega)=-\left(a_{1} \omega+a_{3} \omega^{3}\right)-i\left(\Gamma+a_{2} \omega^{2}+a_{4} \omega^{4}\right)$ in the region $-1<\omega<1$. We find that the set of parameters $\left(\Gamma, a_{1}, a_{2}, a_{3}, a_{4}\right)$ equals $(5.70895,2.28419,1.01368$, $1.15838,0.580893)$ at $T=1 / 4,(2.70929,9.54693$, $5.49701,-2.19662,5.38239)$ at $T=1 / 20$, and $(2.061$ $42,11.8766,16.49,-10.2929,-6.21199)$ at $T=1 / 60$, respectively. As the temperature decreases, the spectral function starts having a dip at $\omega=0$ close to the points $\boldsymbol{k}=(0, \pm \pi)$ and $( \pm \pi, 0)$ along the (unhybridized) Fermi surface line $\epsilon_{c k}=\epsilon_{f k}=0$, as shown in Figs. 3(d)-3(f).
This transition from a peak to a dip at $\omega=0$ marks the boundaries of the Fermi arc.

Summary.-In this work, we studied the heavy-fermion systems using PAMs. We demonstrated that the difference in electron correlations between the $c$ and $f$ electrons in PAMs naturally results in two distinct lifetimes in the nonHermitian quasiparticle Hamiltonian. This, combined with a $d$-wave hybridization gap, leads to the appearance of the EPs in the Hamiltonian and Fermi arcs in the electron spectral function, provided the temperature is sufficiently low. This result should be contrasted with the $s$-wave hybridization, where the Fermi arcs and the EPs are absent. Our DMFT calculation with a numerically exact continuoustime quantum Monte Carlo solver confirmed our findings. These unusual Fermi arcs due to the EPs can be observed by ARPES, which measures electron spectral function. Our results are qualitatively applicable to other systems. In a two-dimensional Kondo system with the $p$-wave hybridization, $V_{\boldsymbol{k}}=\boldsymbol{v}_{\boldsymbol{k}} \cdot \boldsymbol{\sigma}$ with $\boldsymbol{v}_{\boldsymbol{k}}=\left(\sin k_{x}, \sin k_{y}\right)$, the cross-shaped Fermi arcs appear around points $\boldsymbol{k}=( \pm \pi, 0)$ and $(0, \pm \pi)$. In a three-dimensional system, the Fermi arc becomes the three-dimensional Fermi arc defined by the surface with the boundaries in momentum space, which will be studied elsewhere.

Y. N. would like to thank H. Shen for helpful discussions and comments. The work at MIT was supported by DOE Basic Energy Sciences, Division of Materials Sciences and Engineering under Award No. DE-SC0010526. The calculations were performed by the supercomputing system SGI ICE X at the Japan Atomic Energy Agency. Y. N. was 
partially supported by JSPS KAKENHI Grants No. $15 \mathrm{~K} 00178$ and No. 18K03552, the "Topological Materials Science" (No. JP16H00995 and No. 18H04228) KAKENHI on Innovative Areas from JSPS of Japan. Y. Q. was partially supported by NSFC under Grant No. 11874115. V. K. was supported by the Quantum Materials program at LBNL, funded by the U.S. Department of Energy under Contract No. DE-AC0205CH11231. L.F. was partly supported by the Simons Investigator award from the Simons Foundation.

Y. N. and Y. Q. contributed equally to this work.

Note added.- Recently, we learned about the related works Refs. [33,34].

[1] A. H. Castro Neto, F. Guinea, N. M. R. Peres, K. S. Novoselov, and A. K. Geim, The electronic properties of graphene, Rev. Mod. Phys. 81, 109 (2009).

[2] P. M. Ostrovsky, I. V. Gornyi, and A. D. Mirlin, Electron transport in disordered graphene, Phys. Rev. B 74, 235443 (2006).

[3] G. Li, Z. Xiang, F. Yu, T. Asaba, B. Lawson, P. Cai, C. Tinsman, A. Berkley, S. Wolgast, Y. S. Eo, D.-J. Kim, C. Kurdak, J. W. Allen, K. Sun, X. H. Chen, Y. Y. Wang, Z. Fisk, and Lu Li, Two-dimensional Fermi surfaces in Kondo insulator $\mathrm{SmB}_{6}$, Science 346, 1208 (2014).

[4] B.S. Tan, Y.-T. Hsu, B. Zeng, M. Ciomaga Hatnean, N. Harrison, Z. Zhu, M. Hartstein, M. Kiourlappou, A. Srivastava, M. D. Johannes, T. P. Murphy, J.-H. Park, L. Balicas, G. G. Lonzarich, G. Balakrishnan, and S. E. Sebastian, Unconventional Fermi surface in an insulating state, Science 349, 287 (2015).

[5] H. Liu, M. Hartstein, G. J Wallace, A. J Davies, M. Ciomaga Hatnean, M. D. Johannes, N. Shitsevalova, G. Balakrishnan, and S. E. Sebastian, Fermi surfaces in Kondo insulators, J. Phys. Condens. Matter 30, 16LT01 (2018).

[6] Z. Xiang, Y. Kasahara, T. Asaba, B. Lawson, C. Tinsman, L. Chen, K. Sugimoto, S. Kawaguchi, Y. Sato, G. Li, S. Yao, Y. L. Chen, F. Iga, J. Singleton, Y. Matsuda, and L. Li, Quantum oscillations of electrical resistivity in an insulator, Science 362, 65 (2018).

[7] B. Keimer, S. A. Kivelson, M. R. Norman, S. Uchida, and J. Zaanen, From quantum matter to high-temperature superconductivity in copper oxides, Nature (London) 518, 179 (2015).

[8] A. V. Chubukov, M. R. Norman, A. J. Millis, and E. Abrahams, Gapless pairing and the fermi arc in the cuprates, Phys. Rev. B 76, 180501 (2007).

[9] T. Senthil and P. A. Lee, Synthesis of the phenomenology of the underdoped cuprates, Phys. Rev. B 79, 245116 (2009).

[10] Y. Yamaji and M. Imada, Composite-Fermion Theory for Pseudogap, Fermi Arc, Hole Pocket, and Non-Fermi Liquid of Underdoped Cuprate Superconductors, Phys. Rev. Lett. 106, 016404 (2011).

[11] P. A. Lee, Amperean Pairing and the Pseudogap Phase of Cuprate Superconductors, Phys. Rev. X 4, 031017 (2014).
[12] H. Shen, B. Zhen, and L. Fu, Topological Band Theory for Non-Hermitian Hamiltonians, Phys. Rev. Lett. 120, 146402 (2018).

[13] V. Kozii and L. Fu, Non-Hermitian topological theory of finite-lifetime quasiparticles: Prediction of bulk Fermi arc due to exceptional point, arXiv:1708.05841.

[14] M. Papaj, H. Isobe, and L. Fu, Nodal arc of disordered Dirac fermions and non-Hermitian band theory, Phys. Rev. B 99, 201107(R) (2019).

[15] T. Kato, Perturbation Theory of Linear Operators (Springer, Berlin, 1966).

[16] P. Coleman, Many-Body Physics: From Kondo to Hubbard (Forschungszentrum, Julich, 2015), Chapter 1, 1.1-1.34.

[17] H. Tsunetsugu, M. Sigrist, and K. Ueda, The ground-state phase diagram of the one-dimensional Kondo lattice model, Rev. Mod. Phys. 69, 809 (1997).

[18] G. Nakamoto, T. Takabatake, H. Fujii, A. Minami, K. Maezawa, I. Oguro, and A. A. Menovsky, Crystal growth and characterization of the Kondo semimetal CeNiSn, J. Phys. Soc. Jpn. 64, 4834 (1995).

[19] H. Ikeda and K. Miyake, A theory of anisotropic semiconductor of heavy fermions, J. Phys. Soc. Jpn. 65, 1769 (1996).

[20] M. Dzero, K. Sun, V. Galitski, and P. Coleman, Topological Kondo Insulators, Phys. Rev. Lett. 104, 106408 (2010).

[21] Y. Zhong, Y. Liu, and H.-G. Luo, Topological phase in 1D topological Kondo insulator: $\mathrm{Z}_{2}$ topological insulator, Haldane-like phase and Kondo breakdown, Eur. Phys. J. B 90, 147 (2017).

[22] See Supplemental Material at http://link.aps.org/ supplemental/10.1103/PhysRevLett.125.227204, which includes Refs. [23,24], for the technical details of the perturbation analysis and the DMFT calculation.

[23] H. Schweitzer and G. Czycholl, Second order U-perturbation approach to the Anderson lattice model in high dimensions, Solid State Commun. 69, 171 (1989).

[24] H. Schweitzer and G. Czycholl, Self-consistent U-perturbation treatment of the Anderson lattice model in high dimensions, Physica (Amsterdam) 163B, 415 (1990).

[25] A. Amaricci, G. Sordi, and M. J. Rozenberg, NonFermi-Liquid Behavior in the Periodic Anderson Model, Phys. Rev. Lett. 101, 146403 (2008).

[26] G. Sordi, A. Amaricci, and M. J. Rozenberg, MetalInsulator Transitions in the Periodic Anderson Model, Phys. Rev. Lett. 99, 196403 (2007).

[27] A. Amaricci, L. de' Medici, and M. Capone, Mott transitions with partially filled correlated orbitals, Europhys. Lett. 118, 17004 (2017).

[28] A. Amaricci, L. de' Medici, G. Sordi, M. J. Rozenberg, and M. Capone, Path to poor coherence in the periodic Anderson model from Mott physics and hybridization, Phys. Rev. B 85, 235110 (2012).

[29] K. Yamada and K. Yosida, Fermi liquid theory on the basis of the periodic Anderson Hamiltonian, Prog. Theor. Phys. 76, 621 (1986).

[30] P. Werner and A. J. Millis, Hybridization expansion impurity solver: General formulation and application to Kondo lattice and two-orbital models, Phys. Rev. B 74, 155107 (2006). 
[31] Y. Nagai, S. Hoshino, and Y. Ota, Critical temperature enhancement of topological superconductors: A dynamical mean-field study, Phys. Rev. B 93, 220505 (2016).

[32] L. Huang, Y. Wang, Z. Yang Meng, L. Du, P. Werner, and X. Dai, iQIST: An open source continuous-time quantum Monte Carlo impurity solver toolkit, Comput. Phys. Commun. 195, 140 (2015).
[33] T. Yoshida, R. Peters, and N. Kawakami, Non-Hermitian perspective of the band structure in heavy-fermion systems, Phys. Rev. B 98, 035141 (2018).

[34] Y. Michishita, T. Yoshida, and R. Peters, Relationship between exceptional points and the Kondo effect in $f$-electron materials, Phys. Rev. B 101, 085122 (2020). 